\title{
Physical activity to prevent obesity in young children: cluster randomised controlled trial
}

\author{
John J Reilly, Louise Kelly, Colette Montgomery, Avril Williamson, Abigail Fisher, John H McColl, Rossella Lo Conte,
} James Y Paton, Stanley Grant

\begin{abstract}
Objective To assess whether a physical activity intervention reduces body mass index in young children.

Design Cluster randomised controlled single blinded trial over 12 months.

Setting Thirty six nurseries in Glasgow, Scotland.

Participants 545 children in their preschool year, mean age 4.2 years (SD 0.2) at baseline.

Intervention Enhanced physical activity programme in nursery (three 30 minute sessions a week over 24 weeks) plus home based health education aimed at increasing physical activity through play and reducing sedentary behaviour.

Main outcome measure Body mass index, expressed as a standard deviation score relative to UK 1990 reference data. Secondary measures were objectively measured physical activity and sedentary behaviour; fundamental movement skills; and evaluation of the process.

Results Group allocation had no significant effect on the primary outcome measure at six and 12 months or on measures of physical activity and sedentary behaviour by accelerometry. Children in the intervention group had significantly higher performance in movement skills tests than control children at six month follow-up $(\mathrm{P}=0.0027 ; 95 \%$ confidence interval 0.3 to 1.3 ) after adjustment for sex and baseline performance.

Conclusions Physical activity can significantly improve motor skills but did not reduce body mass index in young children in this trial.

Trial registration Current Controlled Trials

ISRCTN36363490.
\end{abstract}

\section{Introduction}

Obesity in children has increased dramatically in recent years. ${ }^{1}$ It has adverse health consequences, ${ }^{2}$ and there is an urgent need for population based interventions aimed at prevention. ${ }^{34}$ Systematic reviews have reported a dearth of high quality evidence from randomised controlled trials: most older intervention studies were short term, often underpowered, and had other weaknesses such as failure to include a control group. ${ }^{3}$ More recent interventions have usually been unsuccessful. ${ }^{5}$ Only a single long term randomised controlled trial reported as being of high quality in systematic reviews found benefits to the intervention (attributed to reduced time spent watching television). ${ }^{6}$ Despite the need for trials in obesity prevention in children, a systematic review to the end of 2003 identified only six ongoing trials, most of which were focused on adolescent girls from minority groups in the United States. ${ }^{7}$

In 2001 in Scotland at least 10\% of children aged 4-5 and $20 \%$ of children aged 11-12 were obese (body mass index $\geq 95$ th centile). ${ }^{8}$ Children in Scotland establish a physically inactive lifestyle before school entry. ${ }^{9}$ As many young children now attend preschool education ( $>90 \%$ in Scotland), the nursery provides population based opportunities for prevention of obesity. In a pilot study we found that an enhanced physical activity programme in nursery was a promising means of preventing obesity. ${ }^{10}$ We therefore tested the hypothesis that a physical activity intervention would reduce body mass index, expressed as a standard deviation score. We carried out a cluster randomised controlled trial to avoid contamination between intervention and control participants. Design, conduct, and reporting followed guidance from the CONSORT statement on cluster randomised controlled trials. ${ }^{11}$

\section{Participants and methods}

\section{Nurseries and children}

In 2002 we invited 124 nurseries to participate in the movement and activity Glasgow intervention in children (MAGIC) trial. Eligible nurseries had at least 12 children in their preschool year. We randomly selected 36 of the 104 nurseries willing to participate. To ensure comparability of intervention and control groups, nurseries were stratified and pairs of nurseries randomly selected from the same stratum, one randomly allocated to intervention and one to control. Stratification was carried out simultaneously according to three characteristics that might have affected the intervention or study outcomes: type of nursery (school, class, extended day, private sector); size of nursery (area and number of children); and socioeconomic status of the area. All families with children in their preschool year attending the 36 nurseries were invited to participate. Parents gave informed written consent to participation

\section{Intervention}

The intervention had nursery and home based elements and is described in detail elsewhere (www.gla.ac.uk/developmental/ research/activities/Exercise\%20\&\%20Metabolism/Magic/ index.html).

Nursery element-The nursery based element was an enhanced physical activity programme consisting of three 30 minute sessions of physical activity each week over 24 weeks. To deliver the intervention two members of staff from each intervention nursery attended three training sessions. A researcher unblinded to allocation (AW) carried out a 
monitoring visit to assess implementation. The nursery based element of the intervention was intended to increase levels of physical activity and children's fundamental movement skills ${ }^{10}$ and meet the requirements of the "physical development and movement" component of the nursery curriculum in Scotland. Nurseries experience several barriers to meeting this curriculum requirement, including lack of space, and limited competence of staff in physical education. ${ }^{12}{ }^{13}$ The nursery element of the intervention was also intended to be inexpensive and therefore generalisable (capital cost <£200, €297, \$377).

Home element-The home based element of the intervention had two parts: each participating family received a resource pack of materials costing $£ 16$ (€24, \$30), with guidance on linking physical play at nursery and at home, and two simple health education leaflets (one on opportunities for increasing physical activity, summarising our recent evidence that levels of physical activity in preschool children in Glasgow were low'; the second encouraged families to seek opportunities to reduce the time spent watching television). For six weeks during the intervention, each intervention nursery also displayed posters focused on increasing physical activity through walking and play.

Control group -In the control group, nurseries continued with their usual curriculum and headteachers agreed not to enhance their physical development and movement curriculum.

\section{Objective and outcome measures}

We tested the efficacy of the intervention by comparing nurseries allocated to intervention with those in the control group at six months after the start of the intervention (when all children were still in their final year at nursery) and 12 months after the start (when 99\% of children had gone to school). All primary and secondary outcomes were measured less than one week apart in pairs of nurseries, and outcome data were analysed and presented at the cluster (nursery) and child level.

Primary outcome measure-Our primary outcome was body mass index expressed as a standard deviation score. ${ }^{14}$ This was calculated at baseline and at six months (mean 24 weeks, SD 2) and 12 months after the start of the intervention (mean 52 weeks, SD 4). To obtain the score LK, who was blinded to group allocation, measured height to $0.1 \mathrm{~cm}$ and weight to $0.1 \mathrm{~kg}$ in duplicate using a portable stadiometer (Leicester Height Measure, Child Growth Foundation, London) and portable scales (TANITA 300GS, Cranlea, Birmingham) with children in light indoor clothing and no shoes.

Secondary outcome measures-We measured habitual levels of physical activity and sedentary behaviour objectively over six days with accelerometry at baseline and at six months ${ }^{15-17}$ using the CSA/MTI WAM-7164 accelerometer (Manufacturing Technology, Fort Walton Beach, FL, USA). Activity data were summarised as total physical activity (accelerometer count per minute) and proportion of waking hours in moderate or vigorous physical activity (accelerometer count $>3200$ per minute) ${ }^{15}{ }^{17}$ and in sedentary behaviour (no trunk movement; accelerometer count $<1100$ per minute). ${ }^{16}$ Because accelerometers and staff time were limited, in nurseries with more than 15 participating children we randomly selected up to 15 children per nursery for accelerometry. We objectively assessed performance in fundamental movement skills at baseline and six months using the movement assessment battery, which has high validity and reliability in preschool children. ${ }^{17}$ The battery provides a global motor skills score of $0-15$, which is a composite of performance in jumping, balance, skipping, and ball exercises. AF carried out all assessments and was blinded to group allocation.

\section{Sample size and power}

We originally intended to recruit a sample large enough to detect a reduction in standard deviation score of 0.25 with power $80 \%$ at a significance of $5 \%$. Making conservative assumptions about the variance of the paired difference in the standard deviation score, the correlation within nurseries (and hence the loss of efficiency because of clustering), and the attrition rate at 12 months, we set out to recruit at least 400 children from at least 30 nurseries. As we were able to exceed these numbers, post hoc analysis suggests we had power of $80 \%$ to detect a reduction in score of just 0.125 .

\section{Sequence generation and blinding}

All 36 participating nurseries were allocated to group in advance in one operation with stratified random sampling. Allocations were concealed by carrying out randomisation of the 36 nurseries at the same time and informing the liaison researcher and nurseries together.

The researchers who made the outcome measures were blinded to nursery allocation with the exception of the statistician who carried out the allocation (JHM) and the contact between the research team and nurseries (AW).

\section{Statistical analysis and evaluation}

We used multi-level (or hierarchical) modelling for all statistical analysis (MLwiN version 1.10), the iterative generalised least squares method for model fitting, and Wald tests to obtain $\mathrm{P}$ values. We analysed and compared baseline and later results using two level models, level 1 being the individual child and level 2 the nursery (cluster). To achieve approximate normality of errors at both levels, we transformed mean accelerometry count in counts per minute and proportion of time spent in moderate or vigorous physical activity by taking the natural logarithm. The modelling for each variable at baseline began by entering the random effects at nursery and child level (which are required because of the cluster design) along with five fixed effects: an "intercept" term, a slope with respect to age (years), a slope with respect to date of baseline activity measurement (days from start of study), and "dummy" variables for female sex and intervention group. For all the outcome variables that were not derived from measurements of physical activity we introduced a sixth term-namely, a slope with respect to log counts per minute. The modelling of the fixed effects proceeded in a backward stepwise manner until we obtained a final model in which all fixed effects were significant. $P$ values were obtained for retaining the effect in the model, at the point at which it was a candidate for removal; this is in the final model for the effects that are significant, but in an intermediate model (not shown) for the other effects. For the random effects, variance components, and their estimated standard errors are listed. We calculated the intraclass correlation to compare the variation between clusters to the total variation; this is measured on a scale from 0 to 1 , with a value close to 0 indicating that the clusters were all "similar."

The modelling of follow-up data at six and 12 months proceeded in a similar manner, with the follow-up measurement itself (rather than the difference between follow-up and baseline) being used as the response. The fixed effects included an "intercept," slopes with respect to the corresponding measurement at baseline and age at measurement, and "dummy" variables for female sex and intervention group. In models where the response was not derived from physical activity, we included a slope with respect to $\log$ (mean counts per minute) at six months as a further fixed effect. Where the effects of a "dummy" variable and slope were both significant, the interaction term between them was considered for the final additive model as another 


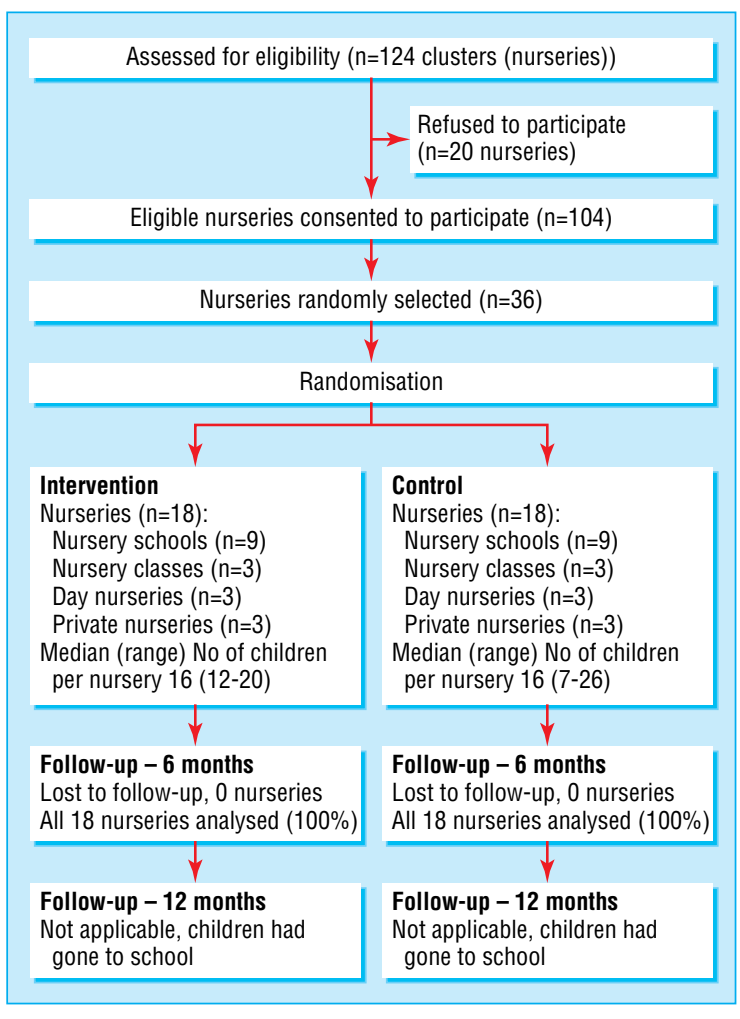

Fig 1 Flow of nurseries though study (study cluster)

fixed effect. In no case was such an interaction significant at the usual 5\% significance level.

We assessed the process of implementation of the intervention by requesting that nurseries record each session of physical activity delivered and attendance by children. We also ensured that nursery staff distributed home based equipment and educational materials to all participating families in the intervention group.

\section{Results}

Thirty six nurseries and 545 children entered the trial. Consent was obtained for the participation of 581 children, but 36 were not available because of absence $(n=29)$ or non-compliance $(\mathrm{n}=7)$. Table 1 shows characteristics of the children. Figures 1 and 2 show the flow of nurseries and children through the trial. All 36 participating nurseries remained in the trial to its completion. Of the 545 children entered at baseline, 481 (88\%) were available at the six month follow-up (while still in nursery) and $504(93 \%)$ were available at the 12 month follow-up, when they were attending 153 different primary schools.

We tried to measure physical activity and sedentary behaviour in 482 children at baseline, and accelerometry was successful in 424 . We attempted six month accelerometry in only the 424 children with accelerometry at baseline, of whom 355 were available, with successful (six day) accelerometry achieved in 285. We obtained data on fundamental movement skills in 489 $(90 \%)$ children at baseline and $420(86 \%$ of the children measured at baseline, $77 \%$ of entire sample) at six months.

\section{Outcomes, estimation, and evaluation}

From the modelling of baseline data, sex was the only fixed effect to enter the model for baseline standard deviation score, the mean score being 0.20 (95\% confidence interval 0.03 to 0.36 , $\mathrm{P}=0.02$ ) lower for girls than for boys.

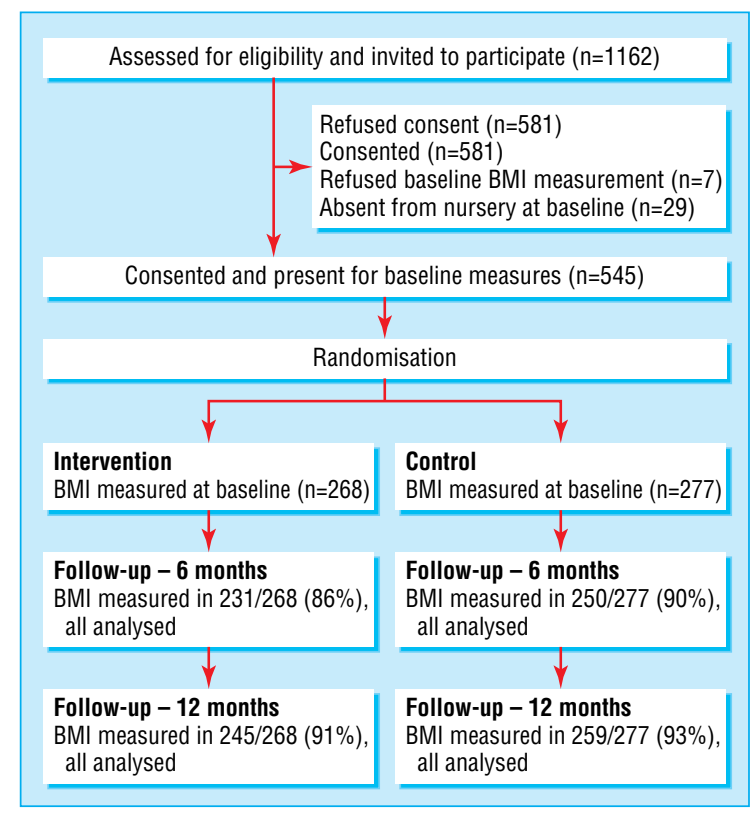

Fig 2 Flow of individual participants through study with primary outcome measure (body mass index (BMI))

Table 2 shows summary statistics for outcome variables at follow-up. The intraclass correlations were $\leq 0.11$, indicating less clustering of final results than expected. The correlation between measurements at baseline and six months was so high that we included the baseline measurement in the final model for every outcome variable.

Group (intervention $v$ control) was not a significant effect in the model for standard deviation score at six months $(\mathrm{P}=0.87)$ or at 12 months $(\mathrm{P}=0.90)$ nor was any other fixed effect significant at either time point. Group was not significant for modelling log counts per minute $(\mathrm{P}=0.18)$ or percentage of time spent sedentary $(\mathrm{P}=0.08)$ but was marginally significant for $\log$ percentage time in moderate or vigorous physical activity (the mean value being greater in the control nurseries by $0.1,0.0$ to $0.2, \mathrm{P}=0.05)$. In modelling the change in score for fundamental movement skills we found that girls improved more than boys, the average difference in improvement being 0.7 units ( 0.3 to 1.1 , $\mathrm{P}=0.001)$. There was a group effect for fundamental movement skills: children in the intervention nurseries improved their movement skills significantly more than children in the control nurseries, the average difference in improvement being 0.8 units (0.3 to 1.3 units).

At the nursery level, $83 \%$ of prescribed sessions of the physical activity programme were actually offered. At the level of the child, $71 \%$ of prescribed sessions were attended (lower quartile $57 \%$, upper quartile $81 \%$ ).

\section{Discussion}

Despite rigorous implementation, we found no significant effect of the intervention on physical activity, sedentary behaviour, or body mass index. Our results add considerably to the evidence base on prevention of childhood obesity because of the paucity of research in children and the serious limitations in study design that affected most previous interventions. ${ }^{34}{ }^{40}$ Our intervention was intended to alter physical activity and sedentary behaviour, not diet. Interventions that have focused intensively on modification of just one or two behaviours have generally been the most promising, ${ }^{4}{ }^{10}$ and reduced physical activity or increased seden- 
Table 1 Baseline characteristics of 545 nursery age children according to activity intervention aimed at reducing body mass index (BMI). Figures are means (SD) unless stated otherwise

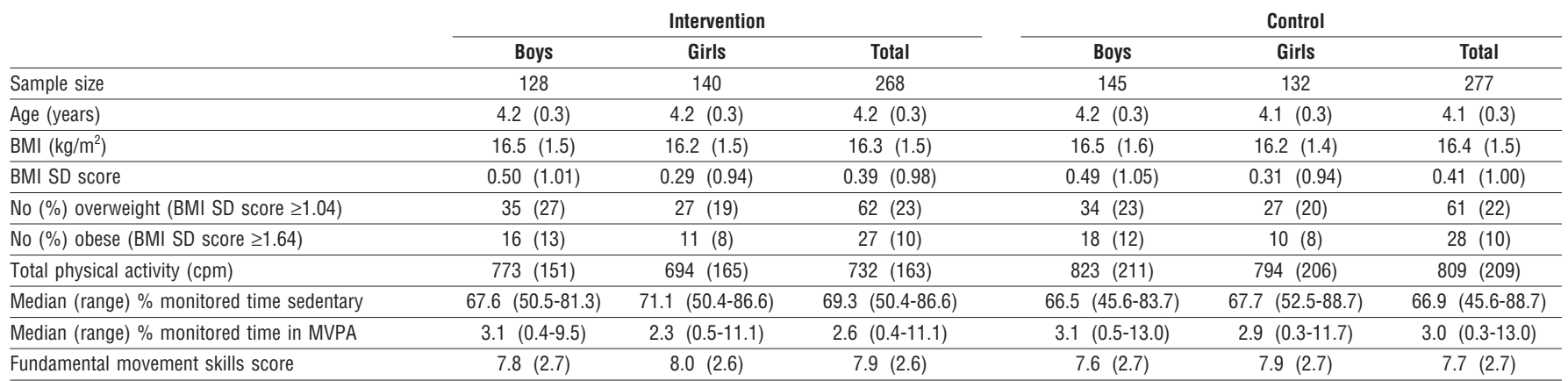

SD score=standard deviation score; $\mathrm{cpm}=$ accelerometry count per minute; MVPA=moderate-vigorous intensity physical activity.

tary behaviour seem to be important in causing and maintaining obesity and are potentially modifiable..$^{10}$

\section{Generalisability and implications}

Our trial was successful from a practical and methodological point of view. In a pilot study over 12 weeks (in four nurseries, with 60 children) we observed significant improvements in physical activity with the intervention, ${ }^{10}$ though we could not replicate these findings in the present study. Quantitatively, implementation of the intervention in our study was apparently good, but the quality of the activity programme might have been higher in the pilot study. Our physical activity programme was delivered by nursery staff (to enhance generalisability), while in the pilot it was delivered by two nursery headteachers. There was evidence of a benefit of the intervention for movement skills. This is an important educational aim and may have other benefits: it might foster an increase in activity levels in future by increasing confidence or ability, or both, in children to carry out physical activity and may have direct effects on body fat content in the long term. ${ }^{17} 19$

To enhance generalisability of our intervention we randomly selected nurseries from those eligible and used an inexpensive intervention that met curriculum requirements and that the pilot study ${ }^{10}$ suggested was learned easily by staff and enjoyed by children.

\section{Strengths and limitations}

Our intervention probably provided an inadequate "dose" of physical activity to have any net impact on overall physical activity (as measured by accelerometry) or the more distal outcome of body mass index. The body mass index is multifactorial, and, while it is acceptable in trials of this kind and practical for large studies, it is not ideal. ${ }^{3410}$ The home based element of the intervention was based largely on the health education model. More behavioural or more extensive interventions with parents ${ }^{20}$ might have been more successful. Our emphasis on using only objective outcome measures provided a rigorous test of the intervention. Some previous studies on obesity prevention that depended on subjective outcome measures have reported benefits to the intervention, but in many cases this was the result of biased self reporting. ${ }^{21}$

\section{Conclusions}

Time in nursery is limited and there is pressure on the curriculum. Successful interventions to prevent obesity in early childhood may require changes not just at nursery, school, and home but in the wider environment. ${ }^{22}{ }^{23}$ Changes in other behaviours, including diet, may also be necessary. Further research is necessary to identify successful and sustainable interventions for prevention of obesity and promotion of physical activity in young children.

We thank Glasgow City Council for their encouragement, advice, and access to nurseries and primary schools. We are immensely grateful to the participating families, nurseries, and schools for their enthusiastic and highly motivated participation. This research was presented in abstract form at the annual meeting of the American College of Sports Medicine in June 2005.

Contributors: JJR was the principal investigator and is guarantor. JJR, AW, JHMcC, SG, and JYP designed the trial with $\mathrm{CM}$, AF, and LK, who helped to refine study design and designed and carried out the outcome measures. $\mathrm{JHMcC}$ and RLC were responsible for statistical analysis and interpretation of the study, and all authors contributed to interpretation. JJR drafted the manuscript, and all authors critically revised it for scientific content and approved the final version.

Funding: British Heart Foundation, Glasgow City Council, and the Caledonian Research Foundation. The pilot study was funded by Sport Aiding Medical Research for Kids (SPARKS).

Competing interests: None declared.

Ethical approval: Yorkhill Hospitals research ethics committee approved the research.

1 Reilly JJ, Dorosty AR. Epidemic of obesity in UK children. Lancet 1999;354:1874-5.

Table 2 Characteristics of nursery age children at follow-up according to activity intervention aimed at reducing body mass index (BMI). Figures are means (SD) unless stated otherwise

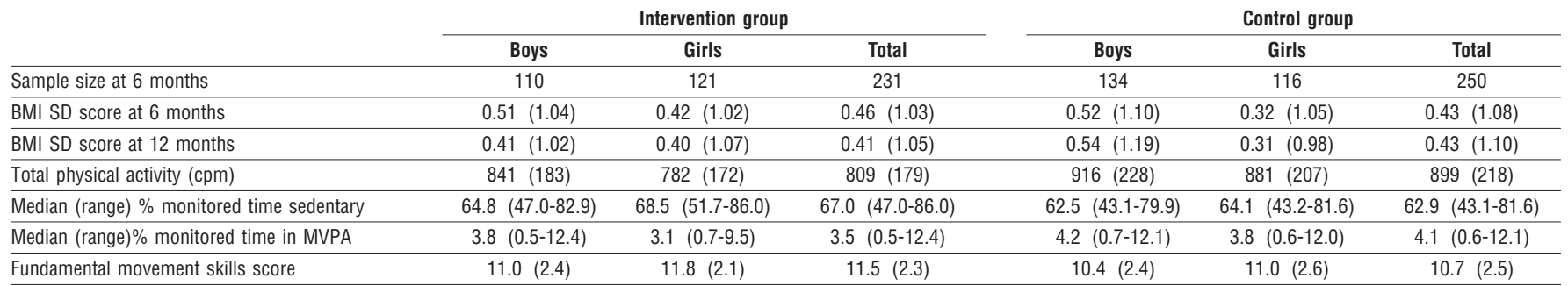

SD score=standard deviation score; $\mathrm{cmm}=$ accelerometry count per minute; MVPA=moderate-vigorous intensity physical activity. 
2 Reilly JJ, Kelnar CJ, Alexander DW, Hacking B, McDowell ZC, Stewart ML, Methven E. Health consequences of obesity: systematic review and critical appraisal. Arch Dis Child 2003;88:748-52.

3 Summerbell CD, Waters E, Edmunds LD, Brown T, Campbell KJ. Interventions for preventing obesity in children. Cochrane Library 2006;(3)CD001871.

4 Reilly JJ, Wilson M, Summerbell C, Wilson D. Obesity diagnosis, prevention, and treatment: evidence-based answers to common questions. Arch Dis Child 2002;86:312-95.

5 Sahota P, Rudolf MCJ, Dixey R, Hill AJ, Barth JH, Cade J. Randomised controlled trial of primary school-based intervention to reduce risk factors for obesity. BMJ 2001;323:1029-32.

6 Gortmaker SL, Peterson K, Wiecha J, Sobol AM, Dixit G, Fox MK, Laird N. Reducing obesity via a school-based interdisciplinary intervention among youth: Planet Health. Arch Pediatr Adolesc Med 1999;151:409-18.

7 Reilly JJ. Obesity prevention in childhood and adolescence: review of systematic reviews. In: Cameron N, Norgan NG, Ellison GTH, eds. 2005. Childhood obesity:strategies for prevention. London: CRC Press, 2005:205-22.

8 NHS quality improvement indicators: childhood obesity in 2001. NHS Scotland, December 2003. www.scot.nhs.uk/indicators (accessed 31 Mar 2005).

9 Reilly IJ, Jackson DM, Montgomery C, Kelly LA, Slater C, Grant S, Paton JY. Total energy expenditure and physical activity in young Scottish children: mixed longitudinal study. Lancet 2004;363:211-2.

10 Reilly JJ, McDowell ZC. Physical activity interventions in the prevention and treatment of paediatric obesity: systematic review and critical appraisal. Proc Nutr Soc 2003;62:611-9.

11 Campbell MK, Elbourne DR, Altman DG, CONSORT Group. CONSORT statement: extension to cluster randomised trials. BMJ 2004;328:702-8.

\section{What is already known on this topic}

Many children are obese, even at preschool age

Preschool children typically have physically inactive

lifestyles

Evidence on appropriate interventions for prevention of obesity in preschool children is lacking

\section{What this study adds}

A physical activity intervention had no effect on body mass index or habitual physical activity

The intervention improved movement skills, which may increase future participation in physical activity or sport

Alternative interventions to prevent obesity in young children are required
12 Williamson A. Factors affecting the development of an appropriate curriculum to promote physical activity in 3-5 year old children in Glasgow pre five establishments. Glasgow: University of Glasgow, 1994. (MEd thesis.)

13 HM Inspectorate of Education. Standards and quality in Scottish pre school education 1997-2001. Edinburgh: Stationery Office, 2002.

14 Cole TJ, Freeman JV, Preece MA. Body mass index reference curves for the UK, 1990. Arch Dis Child 1995;73:25-9.

15 Puyau MR, Adolph AL, Firoz AV, Butte NF. Validation and calibration of activity monitors in children. Obes Res 2002;10:150-7.

16 Reilly JJ, Coyle J, Kelly LA, Burke G, Grant S, Paton JY. An objective method for measurement of sedentary behavior in 3-4 year olds. Obes Res 2003;11:1155-8.

17 Fisher A, Reilly JJ, Montgomery C, Williamson A, Paton JY, Grant S. Fundamental movement skills and habitual physical activity in young children. Med Sci Sports Exerc 2005;37:684-8.

18 Robinson TN. Reducing children's television viewing to prevent obesity: a randomised controlled trial. JAMA 1999:282:1561-7.

19 Okely AD, Booth ML, Chey T. Relationships between body composition and fundamental movement skills among children and adolescents. Res $O$ Exerc Sport 2004:75:238-47.

20 Hardeman W, Griffins, Johnston M, Kinmonth AL, Wareham NJ. Interventions to prevent weight gain: a systematic review of psychological models and behaviour change methods. Int J Obes 2000;24:131-43.

21 Byers T. On the hazards of seeing the world through intervention-colored glasses. Am J Clin Nutr 2003;78:904-5.

22 Koplan JP, Dietz WH. Caloric imbalance and public health policy. JAMA $1999 \cdot 282 \cdot 1579-81$.

23 Swinburn B, Egger G, Razza F. Dissecting obesogenic environments: the development and application of a framework for identifying and prioritising environmental interventions for obesity. Prev Med 1999;29:513-70.

(Accepted 24 August 2006)

doi $10.1136 /$ bmj.38979.623773.55

Division of Developmental Medicine, University of Glasgow, Yorkhill Hospitals, Glasgow G3 8SJ

John J Reilly professor of paediatric energy metabolism

Louise Kelly research assistant

Colette Montgomery postdoctoral research fellow

Abigail Fisher research assistant

James Y Paton reader in paediatric respiratory disease

Glasgow City Council Education Department, Merchant City, Glasgow G1 1HL

Avril Williamson honorary research fellow

Department of Statistics, University of Glasgow, Glasgow G12 8QQ

John H McColl reader in statistics

Rossella Lo Conte research student

University of Glasgow Faculty of Biomedical and Life Sciences, West Medical Building, Glasgow G12 8QQ

Stanley Grant reader in exercise physiology

Correspondence to:J J Reilly jir2y@clinmed.gla.ac.uk 